\title{
Phase diagram of dipolar bosons in two dimensions with tilted polarization
}

\author{
A. Macia, J. Boronat, and F. Mazzanti \\ Departament de Física i Enginyeria Nuclear, Universitat Politècnica de Catalunya, Campus Nord B4-B5, E-08034 Barcelona, Spain
}

(Received 25 July 2014; published 1 December 2014)

\begin{abstract}
We analyze the ground state of a system of dipolar bosons moving in the $X Y$ plane and such that their dipolar moments are all aligned in a fixed direction in space. We focus on the general case where the polarization field forms a generic angle $\alpha$ with respect to the $Z$ axis. We use the path-integral ground-state method to analyze the static properties of the system as both $\alpha$ and the density $n$ vary over a wide range where the system is stable. We use the maximum of the static structure function as an order parameter to characterize the different phases and the transition lines among them. We find that, in addition to a superfluid gas and a solid phase, the system reaches a stripe phase at large tilting angles that is entirely induced by the anisotropic character of the interaction. We also show that the quantum phase transition from the gas to the stripe phase is of second order and report approximate values for the critical exponents.
\end{abstract}

DOI: 10.1103/PhysRevA.90.061601

PACS number(s): 67.85.-d, 03.75.Hh, 05.30.Jp

In recent years, dipolar Bose gases have received much attention. The study of quantum degenerate gases of dipolar species has become one of the most active areas of experimental and theoretical research in the field of ultracold atoms [1-3]. From the theoretical point of view, the anisotropic and long-range character of the interaction makes dipolar systems unique, exhibiting features such as $p$-wave superfluidity in two-dimensional (2D) Fermi gases [4] or roton instability [5-8].

Up to now, little attention has been paid to the 2D case where dipoles are polarized along an arbitrary direction, including the analysis of scattering properties [9] or the superfluid and collapse instabilities of a quasi-two-dimensional gas of dipolar fermions aligned by an external field [4]. Experiments such as those reported in Ref. [10] have also investigated the effect of (quasi-)2D confinement of a cloud of dipolar particles. One remarkable feature induced by the anisotropy of the interaction is the emergence of a stripe phase, which has been predicted to appear in both Bose [7] and Fermi [11-13] systems. Some calculations, in the mean-field approximation, predict the appearance of stripes even in the isotropic case where all dipoles are polarized perpendicular to the plane of movement, although recent Monte Carlo calculations for dipolar fermions arrived at a different conclusion [14]. In Ref. [7] we used path-integral ground-state (PIGS) calculations to reveal the existence of a stripe phase at large densities and polarization angles in 2D dipolar bosonic systems. In this work we extend the previous analysis and discuss the complete phase diagram at zero temperature, characterizing the stripe phase as a function of the density and polarization angle and determining the corresponding solid and stripe transition lines together with the critical exponents.

In previous works we discussed the low-density properties [15] and elementary excitation spectrum [7] of the fully anisotropic 2D dipolar interaction. In this work we extend the analysis and investigate the phase diagram of a $2 \mathrm{D}$ system of bosonic dipoles tilted by an angle $\alpha$ with respect to the normal direction to the plane. The model Hamiltonian describing a system
of $N$ polarized and interacting dipoles is written as

$$
H=-\frac{\hbar^{2}}{2 m} \sum_{j=1}^{N} \nabla_{j}^{2}+\frac{C_{d d}}{4 \pi} \sum_{i<j}^{N}\left[\frac{1-3 \lambda^{2} \cos ^{2} \theta_{i j}}{r_{i j}^{3}}\right]
$$

where $C_{d d}$ is proportional to the square of the (magnetic $\mu$ or electric $d$ ) dipole moment and $\lambda=\sin \alpha$. Polar coordinates $\left(r_{i j}, \theta_{i j}\right)$ describe the separation between the $i$ th and $j$ th particles, respectively. In the following we use dimensionless units obtained from the characteristic dipolar length $r_{0}=$ $m C_{d d} / 4 \pi \hbar^{2}$ and energy $\varepsilon_{0}=\hbar^{2} / m r_{0}^{2}$.

We perform stochastic PIGS [16] calculations in order to build the $T=0$ phase diagram of the system as a function of the dipolar density $n r_{0}^{2}$ and polarization angle $\alpha$. We simulate a finite number of particles $N$ in a box of area $A=N / n r_{0}^{2}$ with periodic boundary conditions. One relevant feature, already present in the two-body problem, is the fact that, in the absence of additional two-body forces, the system can only be stable when the dipolar interaction is strictly non-negative. In this way, there is a critical tilting angle $\alpha_{c} \simeq 0.61$ above which the system collapses because the interaction produces regions where it becomes attractive. It should be noticed that the stability region is so simple to trace because of the tight confinement and the model Hamiltonian used. In a more realistic situation the picture could be different because of inelastic decay processes that can destroy the sample.

The efficiency of the PIGS method is largely enhanced when a suitable variational wave function is used at the end points of the chains representing the interacting particles. Anyway, it is important to remark that estimations of any observable in the PIGS are unbiased with respect to that trial wave function and that, even without it, the results remain unchanged [17]. We have checked that, despite the fact that the interaction is anisotropic, the trial wave function does not need to explicitly incorporate that feature and we have chosen a standard Jastrow product

$$
\Psi_{T}\left(\mathbf{r}_{1}, \mathbf{r}_{2}, \ldots, \mathbf{r}_{N}\right)=\prod_{i<j} f\left(r_{i j}\right)
$$


with the two-body correlation factor given by

$$
f(r)=\left\{\begin{array}{lll}
K_{0}(2 / \sqrt{r}) & \text { if } & r \leqslant R_{M} \\
B \exp \left[-\left(\frac{C}{r}+\frac{C}{L-r}\right)\right] & \text { if } & r>R_{M},
\end{array}\right.
$$

where $B, C$, and $R_{M}$ are constants to be fixed at each density and tilting angle and $L$ is the side of the simulation box. By imposing $f(L / 2)$ to be 1 and $f(r)$ and $f^{\prime}(r)$ to be continuous at $r=R_{M}$, only $R_{M}$ remains unknown and we determine its value through a variational optimization. The two-body correlation factor $f(r)$ built in this way reproduces the exact behavior of the zero-energy solution of the $\alpha=0$ two-body problem at short distances, matched with the box-symmetrized form of a phononic wave function in two dimensions [18].

We know that for $\alpha=0$ the system remains in gas phase up to a freezing density $n r_{0}^{2} \sim 290$, where the system undergoes a first-order phase transition to a triangular solid $[19,20]$. A similar behavior happens when the polarization angle increases, although the transition density changes with $\alpha$. In our simulation we still use Eqs. (2) and (3) at the end points of the PIGS chains in the crystal phase, but in this case the starting configuration corresponds to the sites of the triangular lattice that optimally describe the system This corresponds to an equilateral triangular lattice at $\alpha=0$, but that changes when $\alpha$ increases, squeezing the fundamental triangle of the lattice in such a way that the distance between particles in the direction parallel to the projection of the dipolar moment on the plane is reduced.

In order to characterize the gas-solid transition we use the maximum strength of the static structure factor divided by the number of particles in the simulation as an order parameter, $\eta=S_{\max }(\mathbf{k}) / N$. Figure 1 shows $\eta$ as a function of the density for the polarization angles $\alpha=0.1,0.2,0.3,0.4$ and a different number of particles in the simulation. One can clearly see from the figure that up to a certain density the order parameter is zero, corresponding to a phase where the main peak in $S(\mathbf{k})$

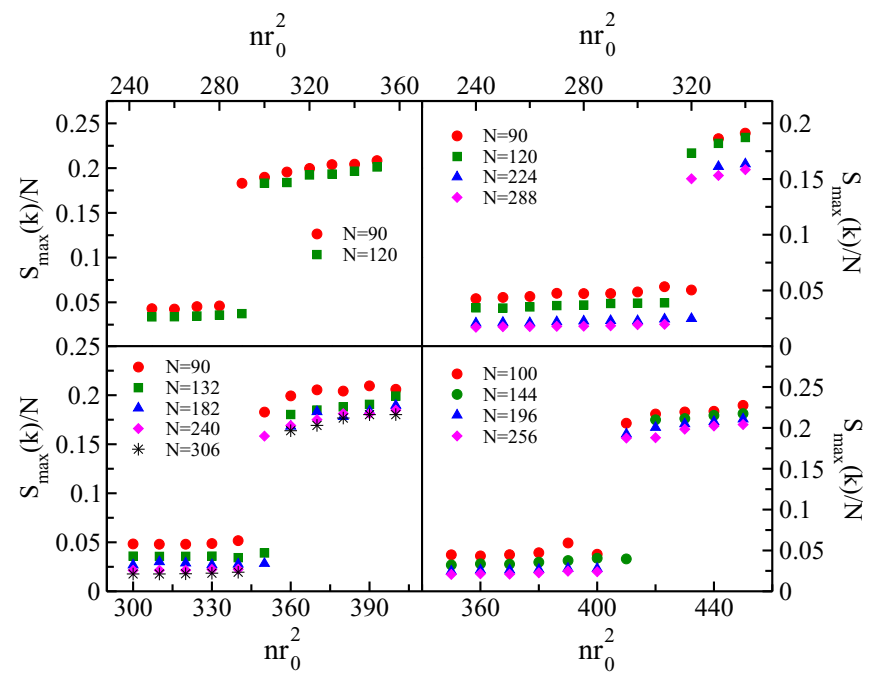

FIG. 1. (Color online) Evolution of the order parameter $S_{\max }(\mathbf{k}) / N$ with the density for $\alpha=0.1$ (top left), $\alpha=0.2$ (top right), $\alpha=0.3$ (bottom left), and $\alpha=0.4$ (bottom right) for an increasing number of particles $N$.
TABLE I. Tilting angle $\alpha$, deformation angle $\gamma$, and transition densities $n_{c} r_{0}^{2}$ of the gas-crystal transition.

\begin{tabular}{lccccc}
\hline \hline$\alpha$ & 0.0 & 0.1 & 0.2 & 0.3 & 0.4 \\
$\gamma$ & $60^{\circ}$ & $60^{\circ}$ & $60^{\circ}$ & $62^{\circ}$ & $64^{\circ}$ \\
$n_{c} r_{0}^{2}$ & $280(20)$ & $290(20)$ & $320(20)$ & $350(20)$ & $410(20)$ \\
\hline \hline
\end{tabular}

does not increase significantly with the number of particles. At higher densities, though, $\eta$ approaches a constant nonzero value, revealing the existence of a main Bragg peak. The discontinuity point indicates the transition density at which crystallization takes place. Table I shows the transition densities for several tilting angles $\alpha$, while $\gamma$ stands for the deformation angle, defined in terms of the primitive vectors of the Bravais lattice

$$
\mathbf{a}_{1}=a \hat{\imath}, \quad \mathbf{a}_{2}=\frac{a}{2}(\hat{\imath}+\hat{\jmath} \tan \gamma),
$$

with $a$ fixed by the density. One sees from the table that the transition density increases with the polarization angle, due to the fact that, overall, the strength of the interaction decreases when $\alpha$ increases. Since the gas-crystal transition is of first order, there are two densities (freezing and melting) defining the coexistence region. In the current case these two densities must be quite close to each other as we have not been able to resolve them from our simulations, as happened also in the isotropic case [19]. Finally, we have checked that the gascrystal transition line is well characterized by a parabolic curve of the form $n_{c} r_{0}^{2}=a+b \sin ^{2} \alpha$, with $\alpha=281.75 \pm 2.75$ and $b=836.41 \pm 34.38$.

By increasing further the tilting angle a new ordered stripe phase appears. The stripe phase is characterized by the emergence of Bragg peaks in $S(\mathbf{k})$ due to the spatial ordering in one direction compared with the gas phase. For this reason we also use here the $\eta$ parameter defined above in order to characterize now the transition from gas to stripes. The top and bottom left panels of Fig. 2 show the evolution of $\eta$

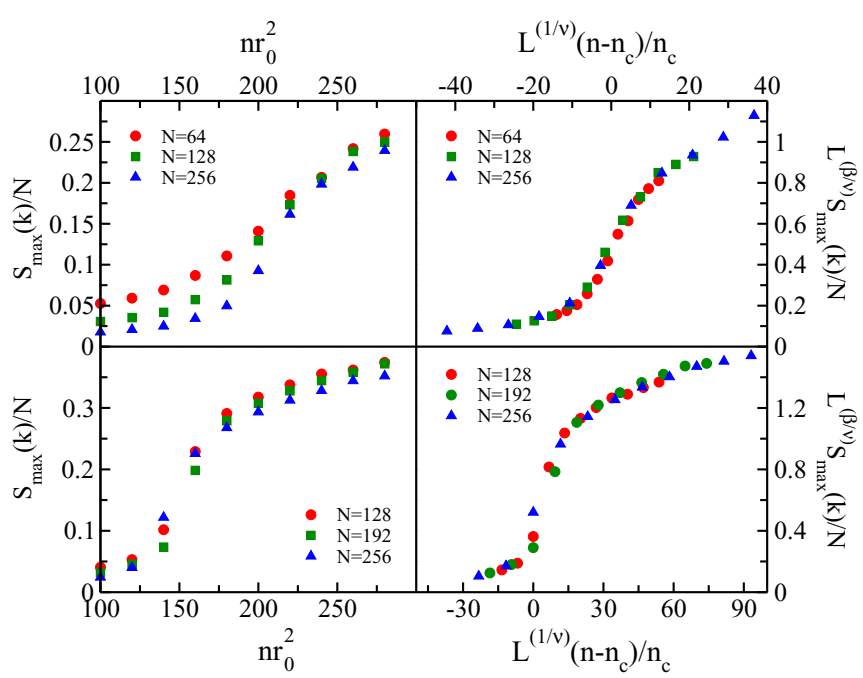

FIG. 2. (Color online) Order parameter $\eta$ for $\alpha=0.54$ and 0.58 as a function of the dipolar density (top and bottom left panels, respectively). 
TABLE II. Tilting angle $\alpha$ and transition densities $n_{c} r_{0}^{2}$ of the gas-stripe transition.

\begin{tabular}{lccccc}
\hline \hline$\alpha$ & 0.52 & 0.54 & 0.56 & 0.58 & 0.60 \\
$n_{c} r_{0}^{2}$ & $260(20)$ & $205(20)$ & $160(20)$ & $140(20)$ & $125(20)$ \\
\hline \hline
\end{tabular}

with the density for $\alpha=0.54$ and 0.58 , respectively, and for different numbers of particles in the simulation. The different behavior when compared with the gas-crystal transition is evident and shows that the transition is in this case continuous. As we are always simulating a finite system in a box with periodic boundary conditions, we use finite-size scaling near the transition point in order to find the critical exponents of this second-order phase transition. We thus employ the following form of a length-scale order parameter:

$$
\eta_{L}(t)=L^{-\beta / v} \tilde{\eta}\left(L^{1 / v} t\right)
$$

corresponding to a system of box side $L$. In this expression $t=$ $\left(n-n_{c}\right) / n_{c}$ is the reduced density around the critical point, while $\nu$ and $\beta$ stand for the critical exponents of the order parameter and the correlation length, respectively, the latter scaling as $t^{-v}$ [21]. In our case we find $v$ and $\beta$ as the optimal values that collapse all curves to a single $\tilde{\eta}$ line. We have found that the best agreement is achieved for $\beta=0.33$ and $\nu=0.63$. The top and bottom right panels in Fig. 2 show the collapse of the data in the respective left panels when these values are used. As it can be seen, the scaling of the data is nicely reproduced, although it is difficult to accurately determine the exact value of the critical exponents from the Monte Carlo data.

An interesting result provided by the finite-size scaling analysis is the fact that the values of the critical exponents do not show a significant dependence on the polarization angle $\alpha$. In this way, for $\alpha \geqslant 0.45$, only the transition density changes when $\alpha$ varies. The values of $v$ and $\beta$ derived from our results are compatible with the classical 3D Ising universality class, $\beta=0.326$ and $v=0.630$, associated with the $\mathrm{U}(1) / Z_{2}$ symmetry breaking arising when the stripes disappear, bearing in mind that the critical behavior of a quantum system in $d$ dimensions is equivalent to that of the corresponding classical system in $d+1$ dimensions [22] The finite-size scaling analysis of the results for different tilting angles allows for the determination of the gas-stripe transition line, which we summarize in Table II. This line turns out to be well fitted by a curve of the form $n_{c} r_{0}^{2}=n_{0} r_{0}^{2}+a \sin ^{2}\left(\alpha-\alpha_{0}\right)$ with $n_{0} r_{0}^{2}=125.59 \pm 3.70, a=18750 \pm 2113$, and $\alpha_{0}=$ $0.6047 \pm 0.0052$.

We end the analysis by describing the high-density and high-polarization-angle region. We have seen that for low and intermediate values of $\alpha$ the system remains in a solid phase at high densities, while stripes appear when $\alpha$ is larger than some critical angle. Consequently, there is a crystal to stripe transition line at an intermediate region. Getting this transition well characterized is difficult from the simulation because the system changes from one high-density ordered phase to another. Still, the different arrangement in each phase can be observed in the static structure factor $S(\mathbf{k})$ : The solid phase is characterized by an infinite number of Bragg peaks located at the characteristic vectors of the reciprocal lattice, while this
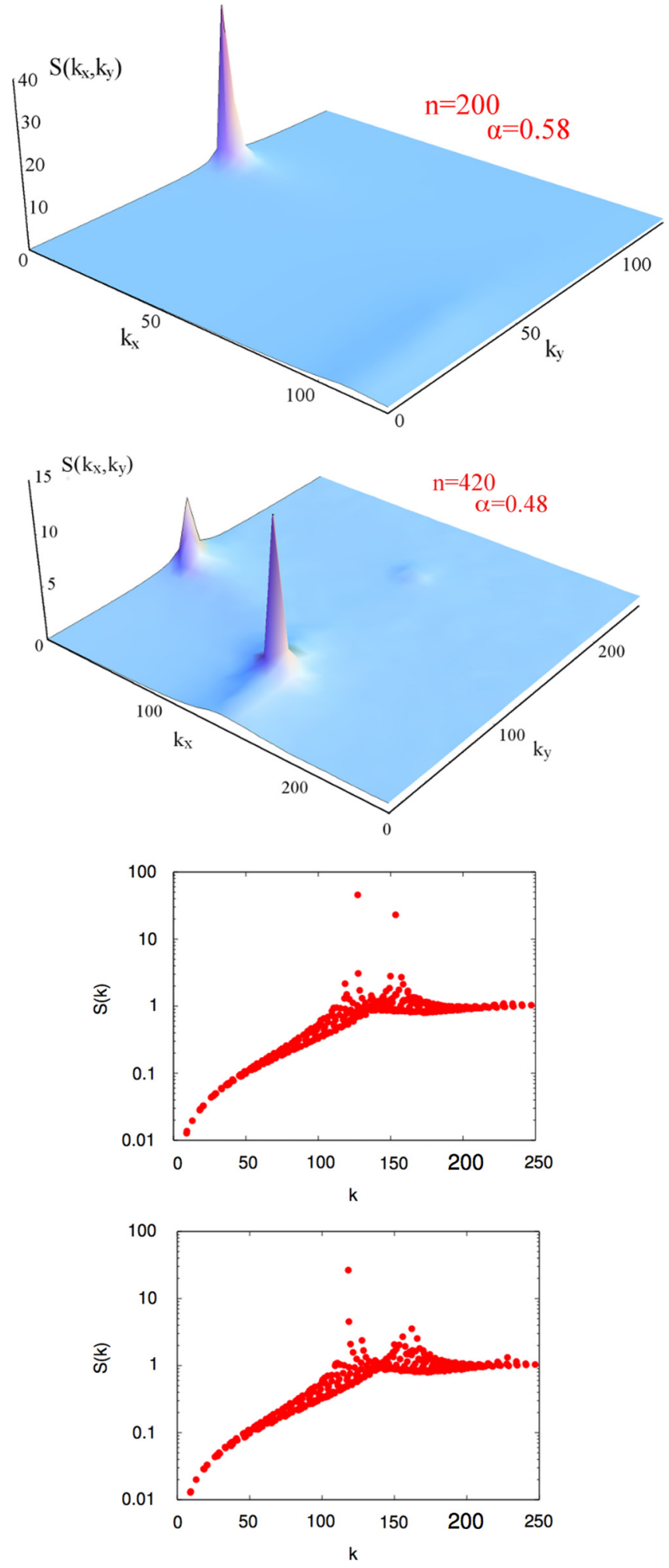

FIG. 3. (Color online) Typical form of the static structure factor $S(\mathbf{k})$ of the system in the stripe and solid phases (two upper panels). The lower panels show the projected $S(\mathbf{k})$ for a density $n r_{0}^{2}=450$ and tilting angles $\alpha=0.48$ (third panel from the top) and $\alpha=0.48125$ (bottom panel). The momenta $k_{x}, k_{y}$, and $k$ are dimensionless quantities expressed in units of $r_{0}^{-1}$. 
TABLE III. Densities and tilting angles corresponding to the solid-stripe transition.

\begin{tabular}{lcccc}
\hline \hline$n_{c} r_{0}^{2}$ & 450 & 480 & 500 & 550 \\
$\alpha$ & $0.4806(1)$ & $0.4819(1)$ & $0.4819(1)$ & $0.4838(1)$ \\
\hline \hline
\end{tabular}

is not the case in the stripe phase, where ordering appears in only one direction.

The upper panels in Fig. 3 show a typical example of the full $S(\mathbf{k})$ in the stripe and solid phases as obtained from our PIGS simulations. As it can be seen, a second peak is clearly resolved in the solid phase in comparison with the stripe phase, where a single Bragg peak associated with the periodicity along the $Y$ axis is visible. In fact, the figure already shows a third peak in the solid phase that has however much less strength but that, together with the other two, put in evidence the structure of the triangular lattice.

In this way, we characterize the stripe to solid phase transition from the emergence of a second Bragg peak in the simulation, absent in the former but present in the latter. The lower panels in Fig. 3 show the emergence of the second Bragg peak for a density $n r_{0}^{2}=450$ with $\alpha=0.48$ (left) and $\alpha=0.48125$ (right). The figure shows the projected $S(\mathbf{k})$ where the full 2D $S(\mathbf{k})$ is represented in a single plot with the magnitude of $\mathbf{k}$ on the $X$ axis. The emergence of the second Bragg peak is quite abrupt and allows finding the solid to stripe transition point at each fixed density. The results obtained with this method are shown in Table III. It is remarkable how steep the curve is, thus indicating that the stripe phase seems to be the stable one up to extremely large densities that are out of reach of our numerical simulations.

An obvious question that arises at this point regards the order of the crystal to stripe phase transition. In this case we have not detected a smooth decay of the second peak of the static structure factor by increasing the polarization angle of the dipoles. On the contrary, the second crystalline Bragg peak suddenly disappears when a slight change in the tilting angle near the transition point is made, thus indicating that the crystal to stripe phase transition is probably of first order.

The full phase diagram of the 2D dipolar Bose system is shown in Fig. 4, where both the simulation points and the transition lines separating the different phases are depicted and separated from the collapse region where the system no longer exists. It is interesting to notice from the phase diagram that there are cuts at constant density in the range $n r_{0}^{2} \in(290,450)$

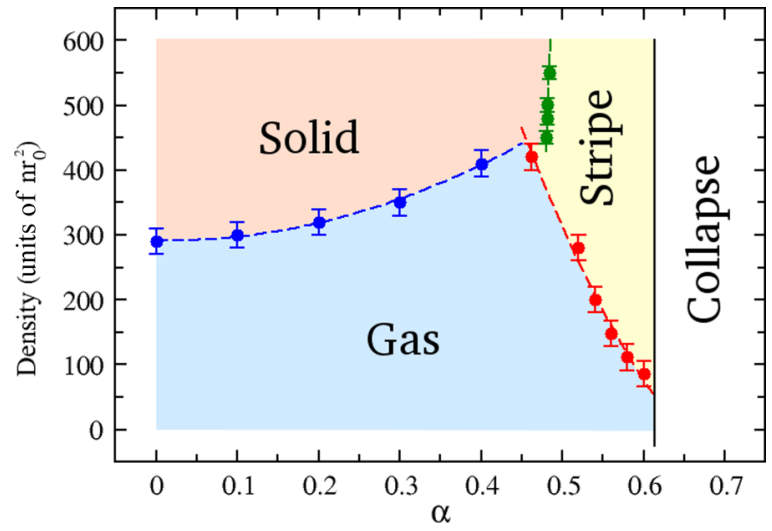

FIG. 4. (Color online) Evolution of the order parameter $S_{\max }(\mathbf{k}) / N$ with the density for $\alpha=0.1$ (top left), $\alpha=0.2$ (top right), $\alpha=0.3$ (bottom left), and $\alpha=0.4$ (bottom right).

where by increasing the polarization angle from $\alpha=0$ all the way to the collapse limit one can find the system in a solid phase, then in a gaseous form, to finally jump into the strip phase. All these changes are due to a delicate balance between the strength of the interaction and its anisotropy, which in some sense compete against each other when the polarization angle increases.

To summarize, using the quantum Monte Carlo method, we have studied the phase diagram of bosonic tilted dipoles in two dimensions relying only on the Hamiltonian of the system. Our results show that at low densities the system is in the gas phase. When the density is increased and the tilting angle is below $\alpha \sim 0.45$ the system undergoes a first-order phase transition and crystallizes. Beyond the critical point at $\alpha \sim 0.45$ a second-order phase transition brings the system from the gas to a stripe phase. The critical exponents of this second-order transition are essentially independent of the polarization angle and are compatible with the 3D Ising universality class within the statistical uncertainty of our simulations. Remarkably, our results show that for large polarization angles the stripe phase can be observed experimentally at densities significantly lower than those required to reach the solid phase and without any optical lattice [23]. Finally, at high densities and large tilting angles the system undergoes a first-order phase transition from the crystal to the stripe phase.

We acknowledge partial financial support from the DGI (Spain) Grant No. FIS2011-25275 and the Generalitat de Catalunya (Spain) Grant No. 2009SGR-1003.
[1] T. Lahaye, C. Menotti, L. Santos, M. Lewenstein, and T. Pfau, Rep. Prog. Phys. 72, 126401 (2009).

[2] M. A. Baranov, Phys. Rep. 464, 71 (2008).

[3] M. A. Baranov, M. Dalmonte, G. Pupillo, and P. Zoller, Chem. Rev. 112, 5012 (2012).

[4] G. M. Bruun and E. Taylor, Phys. Rev. Lett. 101, 245301 (2008).

[5] L. Santos, G. V. Shlyapnikov, and M. Lewenstein, Phys. Rev. Lett. 90, 25, 250403 (2003).

[6] D. H. J. ODell, S. Giovanazzi, and G. Kurizki, Phys. Rev. Lett. 90, 11, 110402 (2003).
[7] A. Macia, D. Hufnagl, F. Mazzanti, J. Boronat, and R. E. Zillich, Phys. Rev. Lett. 109, 235307 (2012).

[8] A. K. Fedorov, I. L. Kurbakov, Y. E. Shchadilova, and Yu. E. Lozovik, Phys. Rev. A 90, 043616 (2014).

[9] C. Ticknor, Phys. Rev. A 84, 032702 (2011).

[10] T. Koch, T. Lahaye, J. Metz, B. Fröhlich, A. Griesmaier, and T. Pfau, Nat. Phys. 14, 218 (2008).

[11] Y. Yamaguchi, T. Sogo, T. Ito, and T. Miyakawa, Phys. Rev. A 82, 013643 (2010).

[12] K. Sun, C. Wu, and S. Das Sarma, Phys. Rev. B 82, 075105 (2010). 
[13] M. M. Parish and F. M. Marchetti, Phys. Rev. Lett. 108, 145304 (2012).

[14] N. Matveeva and S. Giorgini, Phys. Rev. Lett. 109, 200401 (2012).

[15] A. Macia, F. Mazzanti, J. Boronat, and R. E. Zillich, Phys. Rev. A 84, 033625 (2011)

[16] A. Sarsa, K. E. Schmidt, and W. R. Magro, J. Chem. Phys. 113, 1366 (2000).

[17] R. Rota, J. Casulleras, F. Mazzanti, and J. Boronat, Phys. Rev. E 81, 016707 (2010).

[18] L. Reatto and G. V. Chester, Phys. Rev. 155, 88 (1967).
[19] G. E. Astrakharchik, J. Boronat, I. L. Kurbakov, and Yu. E. Lozovik, Phys. Rev. Lett. 98, 060405 (2007).

[20] H. P. Büchler, E. Demler, M. Lukin, A. Micheli, N. Prokof'ev, G. Pupillo, and P. Zoller, Phys. Rev. Lett. 98, 060404 (2007).

[21] H. E. Stanley, Introduction to Phase Transitions and Critical Phenomena (Oxford University Press, Oxford, 1971).

[22] S. Sachdev, Quantum Phase Transitions (Cambridge University Press, Cambridge, 2011).

[23] T. Ohgoe, T. Suzuki, and N. Kawashima, Phys. Rev. A 86, 063635 (2012). 